\title{
Periódicos científicos: critérios de qualidade
}

\section{Scientific periodicals: quality criteria}

\author{
Maria Cecilia Gonzaga Ferreira* \\ Rosaly Favero Krzyzanowski**
}

\begin{abstract}
RESUMO: Traz uma revisão histórica da literatura sobre avaliação de periódicos e qual a metodologia utilizada nessas avaliações. Enfoca a necessidade de se cuidar dos aspectos de conteúdo (mérito) dos periódicos juntamente com os relacionados a forma de apresentação baseada em normas técnicas para que o periódico atinja uma qualidade global. Apresenta um resumo dos aspectos mais importantes das normas para apresentação de periódicos e artigos científicos.
\end{abstract}

DESCRITORES: Publicações periódicas; Normas.

ABSTRACT: This paper presents a historical literature review on the evaluation of periodicals and the methodology employed for their evaluation. It emphasizes the attention that should be given to the contents of the periodicals and their format based on technical standards in order to reach a global quality of the publications. This paper includes a summary of the most important aspects of the technical standards for periodicals and scientific articles.

DESCRIPTORS: Periodicals; Standards.

\section{INTRODUÇÃO}

A publicação de revistas científicas nas diversas áreas do conhecimento humano tem apresentado nas últimas décadas um aumento considerável, conforme podemos verificar no gráfico de Wieers (Gráfico 1).

Esse crescente aumento do número de publicações científicas em diferentes suportes tem preocupado os profissionais que se interessam pela qualidade da informação, sejam eles autores, editores, publicadores, centros de documentação, bibliotecas ou pesquisadores. Acompanhando esse volume de informações, surgiram diversos problemas relacionados às publicações que têm ensejado críticas com relação à qualidade da informação divulgada através dos periódicos, nos âmbito internacional e nacional. Entre essas críticas, pode-se destacar (Krzyzanowski et al. $\left.{ }^{6}, 1991\right)$ :

- irregularidade na publicação e distribuição da revista;

- falta de normalização dos artigos científicos e da revista como um todo;

- problemas ligados à avaliação de conteúdo, tais como: corpo editorial idôneo e processo de "peer review" inadequado.

Com relação às revistas nacionais, pode-se acrescentar ainda:
- pouca penetração da língua portuguesa no âmbito internacional;

- baixo grau de originalidade e novidade dos artigos científicos;

- falta de recursos financeiros.

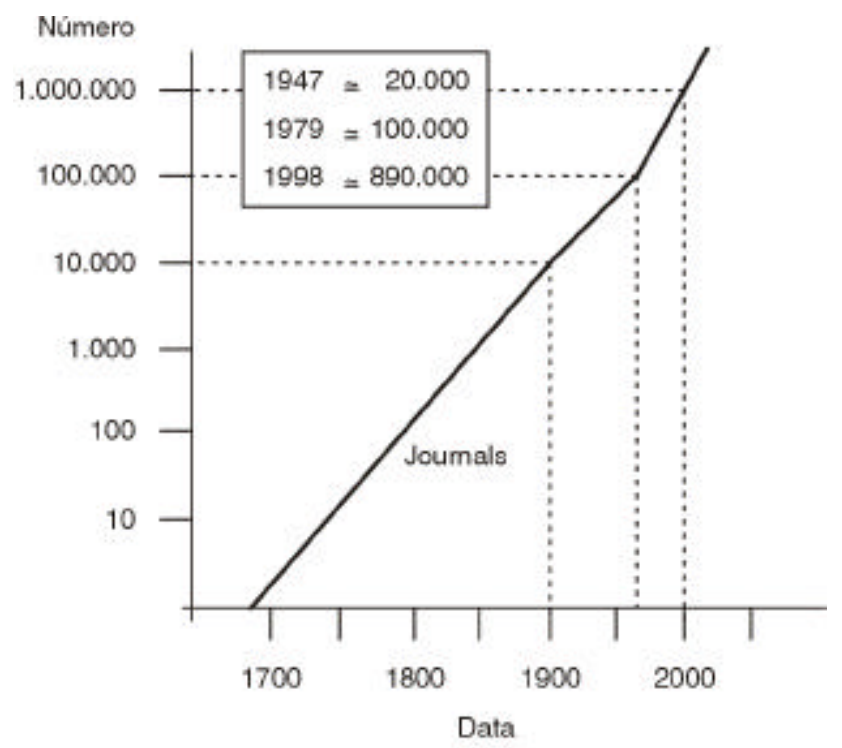

GRÁFICO 1 - Número de revistas científicas correntes em relação a datas. Fonte: Wieers L. A vision on the library of the future. In: Geleijnse H, Grootaers C. Developing the library of the future: the Tiburg experience. Tiburg: Tiburg University; 1994. p.1-11.

\footnotetext{
* Bibliotecária Assessora do Programa Biblioteca Eletrônica - ProBE/FAPESP.

** Bibliotecária Coordenadora Operacional do Programa Biblioteca Eletrônica - ProBE/FAPESP.
} 
Esses fatores negativos, que prejudicam o padrão de qualidade das revistas científicas brasileiras, também têm dificultado a sua aceitabilidade no meio técnico-científico internacional, impossibilitando sua indexação em bases de dados. Sabe-se que algumas dessas bases de dados possuem seus critérios próprios e filtros de qualidade para inclusão dos periódicos. Como conseqüência, a ciência e pesquisa desenvolvidas no Brasil têm a sua visibilidade prejudicada com a baixa divulgação dos periódicos nacionais. Acrescente-se ainda que um segmento de autores nacionais, que pertencem às frentes de pesquisa vem procurando publicar o resultado de seus trabalhos em revistas internacionais.

\section{REVISÃO DE LITERATURA}

A partir de 1960, encontram-se na literatura nacional diversos trabalhos sobre avaliação de periódicos, que refletem a necessidade do estabelecimento de parâmetros para medir a qualidade das informações veiculadas através dos artigos científicos. Entre esses trabalhos, pode-se destacar, cronologicamente, os que se encontram na literatura da área da informação.

- 1964 - a Unesco ${ }^{9}$ cria um modelo de mensuração para avaliação de revistas latino-americanas, que classifica as revistas nas categorias de deficiente a excelente.

- 1968 - Arends ${ }^{1}$, baseada no modelo da Unesco (1964), estabelece critérios relacionados a: apresentação do material, regularidade da publicação, tempo de existência, periodicidade, aceitação de colaboradores de outras instituições, indexação, entre outros.

- 1982 - Braga et al. ${ }^{2}$ apresentam uma proposta de avaliação modificando o modelo da Unesco. O modelo procura analisar aspectos de forma dos periódicos dentro de parâmetros mensuráveis. A cada critério corresponde um número de variáveis e condições para que o periódico obtenha uma pontuação. O número total de pontos que o periódico atinge determina o seu nível de desempenho (muito bom, bom, mediano e fraco). Segundo as autoras do trabalho, "a escolha dos critérios foi norteada por sua aplicabilidade (existência de ferramentas para coleta e análise), pelas características peculiares aos periódicos nacionais - restrições econômicas, tipográficas, etc. $-e$, principalmente, pela validade que conferem ao julgamento de qualidade de suas funções básicas das publicações periódicas, isto é, a função memória - arquivo do conhecimento - e a função disseminação - transmissão ampla de idéias" (Braga $\left.{ }^{2}, 1982\right)$.

- 1985 - Yahn ${ }^{11}$ utilizando o modelo de Braga, introduz modificações e sugere que o resultado deve abranger a avaliação conjunta de mérito (conteúdo dos artigos) e desempenho (forma). O modelo foi aplicado em periódicos na área da Agricultura.

- 1986 - Martins ${ }^{8}$ avalia 224 títulos da área de Ciência e Tecnologia utilizando um formulário que visa verificar itens referentes à normalização, baseados nas normas da ABNT (Associação Brasileira de Normas Técnicas).

- 1991 - Krzyzanowski et al. ${ }^{6}$ deram seqüência ao projeto de avaliação de periódicos científicos brasileiros correntes iniciado em 1988, visando refinar e atualizar o núcleo básico de revistas científicas correntes nacionais nas diferentes áreas do conhecimento definido no primeiro estudo, com o objetivo de subsidiar o programa de apoio financeiro a revistas científicas da FAPESP. A metodologia adotada nos dois estudos (1988 e 1991) teve como princípio a avaliação de mérito das revistas pelos seus pares, mediante parâmetros predefinidos. As revistas foram classificadas de acordo com o seu nivel de relevância em: prioritárias, importantes e de importância relativa.

- 1996 - Castro, Ferreira ${ }^{3}$ realizam na Bireme (Centro Latino-Americano e do Caribe de Informação em Ciências da Saúde), a pedido da OPAS (Organização Pan-Americana da Saúde), a avaliação de 311 periódicos latino-americanos indexados no Medline e na Lilacs (Literatura Latino-Americana e do Caribe em Ciências da Saúde), tentando identificar se as características formais e de divulgação de periódicos podem ser indicativas da qualidade dos mesmos, no processo de seleção de títulos a serem incluídos em bases de dados. O estudo utilizou o modelo de avaliação de $\mathrm{Braga}^{2}$ (1982), com modificações. Com os resultados foi possivel verificar os aspectos de forma dos periódicos que podem prejudicar a sua qualidade e conseqüente indexação em bases de dados internacionais.

- 1997 - É realizada na Bireme uma nova avaliação, a partir do refinamento da anterior, tendo sido modificados e ampliados os itens do formulário que foi utilizado em 1995, principalmente, com relação a análise das instruções aos autores. O estudo teve como objetivo trazer subsí- 
dios para o estabelecimento de critérios de seleção para entrada de periódicos na base de dados SciELO (Scientific Electronic Library Online).

- 1998 - Krzyzanowski, Ferreira ${ }^{7}$, a pedido das agências financiadoras: FINEP (Financiadora de Estudos e Projetos), CNPq (Conselho Nacional de Desenvolvimento Científico e Tecnológico) e FAPESP realizaram uma avaliação de periódicos financiados pelas respectivas agências. O universo estudado foi de 407 títulos. A metodologia utilizada foi desenvolvida para a realização de uma avaliação conjunta de mérito (conteúdo) e desempenho (forma). A avaliação de conteúdo, foi realizada seguindo procedimentos previamente estabelecidos e o formulário foi aplicado por pesquisadores das diferentes áreas do conhecimento humano (avaliação pelos pares) o que permitiu a classificação das revistas em três níveis de relevância: prioritária, importante e de importância relativa. A avaliação de forma (desempenho) aplicada foi baseada no modelo de Braga ${ }^{2}$ (1982), que estabelece critérios de pontuação, determinando o nivel de desempenho (muito bom, bom, mediano e fraco). Os resultados desse estudo demonstraram que o periódico necessita ser avaliado tanto no seu conteúdo quanto a sua apresentação formal para que se possa verificar a sua qualidade global.

- 1999 - Yamamoto ${ }^{12}$ realizaram a partir de uma demanda da Coordenação de Aperfeiçoamento de Pessoal de Nivel Superior (CAPES), uma avaliação de periódicos científicos em Psicologia. O modelo proposto foi baseado na avaliação de Krzyzanowski, Ferreira ${ }^{7}$ (1998), com modificações. Os periódicos foram também classificados de acordo com o seu âmbito em local (periódicos de âmbito restrito de circulação) e nacional (periódicos de circulação maior). As variáveis também foram adaptadas ao âmbito.

\section{Considerações sobre as avaliações}

Todos os estudos realizados refletiram praticamente a mesma preocupação com a qualidade das informações veiculadas através dos periódicos. Constatam-se vários pontos em comum nessas avaliações. Esses parâmetros que mensuraram os periódicos e lhes deram uma classificação estão basicamente relacionados com os aspectos de conteúdo e normalização, que serão abordados a seguir.

\section{CONTEÚDO}

A qualidade do conteúdo de um periódico é que vai determinar o seu mérito. Neste contexto devem ser levados em consideração aspectos relacionados a (Krzyzanowski, Ferreira $\left.{ }^{7}, 1998\right)$ :

- qualidade dos artigos (nível científico; atualidade; identificação com a orientação temática da revista; percentual de artigos originais);

- qualidade do corpo editorial e dos consultores (participação de membros da comunidade nacional e internacional);

- critérios de arbitragem dos textos;

- natureza do órgão publicador (os periódicos devem ser editados de preferência por instituição de ensino, pesquisa e sociedade científica);

- abrangência quanto à origem dos trabalhos (abertura da revista para autores de nível institucional, nacional e internacional);

- difusão da revista (distribuição e divulgação deve ser a mais ampla possivel);

- indexação - a revista deve pleitear a inclusão das bases de dados nacionais e internacionais de acordo com a área de assunto que abrange. Quanto maior o número de bases de dados, maior será a valorização de sua qualidade, produtividade e, inclusa, a difusão indireta da revista.

\section{NORMALIZAÇÃO}

Quanto à normalização o que fica evidente é que os periódicos e seus artigos científicos devem seguir rigorosamente as normas adotadas. Sejam elas as normas da Associação Brasileira de Normas Técnicas (ABNT), do Grupo de Vancouver, da American Psychological Association ou outra. Atualmente, o rigor no seguimento das normas torna-se imprescindivel, pois os sistemas automatizados necessitam que os dados estejam em perfeita sintonia com as normas, para que os computadores possam interpretar eletronicamente os dados.

Visando a orientação de editores são apresentados, resumidamente, aspectos importantes na preparação dos periódicos e seus artigos (Ferrei$\left.\mathrm{ra}^{4,5}, 2001 \mathrm{a} ; 2001 \mathrm{~b}\right)$. Os aspectos abordados são baseados nas normas da ABNT (NBR-6021); NBR-6022 e outras relacionadas. A obtenção das normas e endereço dos escritórios da ABNT podem ser encontrados no site: $h t t p: / / w w w$.abnt.org.br. 


\section{Formato do periódico}

Recomenda-se manter sempre o mesmo. As mudanças só devem ocorrer no início de novos volumes.

\section{Capa}

Deve conter: título do periódico, instituição responsável, ISSN, número do volume, fascículo, mês(es), por extenso, ano de publicação.

\section{Folha de rosto}

Deve conter: legenda bibliográfica, indicação de editor e outros responsáveis pela publicação do periódico, endereço completo, indicação dos responsáveis pela revisão técnica, forma de aquisição e preço de assinatura, relação dos "referees" e fontes indexadoras do periódico.

\section{Legenda bibliográfica}

Deve estar de acordo com a NBR 6026, devendo estar incluída na capa, sumário e páginas do texto. A legenda é um conjunto de dados necessários à identificação de uma publicação periódica, fascículos e artigos de periódicos. Deve conter: título do periódico, local de publicação, número do volume, número do fascículo, número da página inicial e final de cada artigo (nas folhas do texto do artigo), data (mês e ano). Na capa os meses devem ser registrados por extenso.

\section{ISSN}

Todos os periódicos deverão ter o seu ISSN (International Standard Serial Number), que é fornecido pelo IBICT (Instituto Brasileiro de Informação em Ciência e Tecnologia). O ISSN deverá ser colocado na capa, página de rosto e/ou sumário. Orientações sobre o ISSN podem ser obtidos no site: $h t t p: / / w w w . i b i c t . b r$.

\section{Sumário}

Enumeração das seções e/ou artigos de um fascículo. Deve figurar no início do documento. Recomenda-se que o sumário seja na língua do texto e em inglês. O sumário deve estar de acordo com a NBR-6027.

\section{Resumos}

Apresentação concisa dos pontos relevantes de um texto (até 250 palavras). Os resumos permitem ao leitor decidir sobre a necessidade de consulta ao texto original e/ou transmitir informações de caráter complementar. Facilitam também o trabalho dos indexadores de bases de dados. Devem ser prepara- dos, no mínimo, em dois idiomas. Alguns periódicos estão optando, no caso de artigos originais, por resumos estruturados. A norma NBR-6028 orienta sobre a redação e estilo próprio dos resumos.

\section{Unitermos, descritores, palavras-chave}

São termos indicativos do conteúdo dos artigos e devem ser escolhidos, sempre que possivel, utilizando-se vocabulários controlados. São muito importantes para a indexação correta dos artigos em bases de dados e para a futura busca dos artigos por assunto. Os unitermos devem acompanhar os resumos em português e os "abstracts", resumos em inglês. Na área biomédica, recomenda-se usar os "Descritores em Ciências da Saúde - DeCS", vocabulário publicado pela Bireme, sendo uma tradução do vocabulário de assuntos (Mesh) do Medline. O DeCS pode ser consultado no site: http://www.bireme.br.

\section{Referências bibliográficas}

É importantíssimo uniformizar a apresentação das referências, pois isso facilitará a identificação dos trabalhos referenciados, agilizando a sua localização e obtenção. Para elaboração das referências as normas mais utilizadas são as da: International Standard Organization (ISO-690); Grupo de Vancouver (utilizadas pelas revistas internacionais da área biomédica, que adotam essas normas para a preparação de artigos); Norma da American Psychological Association - utilizada na área de humanas, traz exemplos de documentos eletrônicos; NBR-6023, agosto 2000, da ABNT, que está bastante ampliada trazendo inclusive exemplos de referências de documentos eletrônicos, legislação, documentos multimídia, etc. (fotografia, diapositivos, vídeos, transparência, outros).

\section{Citação no texto}

Menção no texto dos artigos de uma informação colhida de outra fonte, pode ser uma paráfrase (citação indireta) ou transcrição direta (entre aspas). É imprescindivel a menção das diversas fontes utilizadas no trabalho. O método adotado para a citação no texto deve ser o adotado pela revista e deve ser seguido em todo o trabalho de forma consistente (NBR-10520).

\section{Instruções aos autores}

Devem ser claras e abrangentes para facilitar a normalização dos artigos, orientar os autores e dar autoridade ao periódico para devolver trabalhos 
que não estejam dentro dos parâmetros da revista. Incluir informações relativas a:

a) objetivo da revista;

b) tipos de idiomas aceitos;

c) critérios utilizados na seleção dos trabalhos (processo de "peer-review");

d) declaração de responsabilidade do autor pelo conteúdo do trabalho e de conflitos de interesse que possam interferir nos resultados;

e) transferência de direitos autorais;

f) indicação da categoria de artigos aceitos e orientação quanto a forma de apresentação de cada tipo (originais, revisão, etc.);

g) orientação geral quanto a forma de envio do material (impressa, disquete, e-mail, etc.);

h) programas e versões aceitas, tipos de fontes;

i) títulos do artigo (em português, inglês, ou outro idioma), devem ser concisos para que identifiquem o assunto a que se refere o artigo;

j) resumos no idioma do texto e em inglês;

k) orientação sobre o estabelecimento de descritores no idioma do texto e em inglês;

1) incluir exemplos de referência bibliográfica de vários tipos de documentos e especificar a norma utilizada;

m) orientação quanto a apresentação de ilustrações, abreviaturas e siglas;

n) filiação completa dos autores (nome completo, instituição a que pertencem, endereço, e-mail, destacando o autor responsável pela troca de correspondência com a revista).

A seguir, são detalhados outros aspectos que reforçam a qualidade da apresentação formal do periódico.

\section{Título do artigo}

Deve ser conciso e completo e indicar o conteúdo do artigo. Não devem ser utilizadas palavras supérfluas.

\section{Regularidade da publicação}

A regularidade da publicação é um dos critérios obrigatórios no processo de avaliação dos periódicos. Os periódicos devem aparecer pontualmente de acordo com a periodicidade estabelecida.

\section{Periodicidade}

É recomendável que o periódico seja no mínimo quadrimestral, e dependendo da área (saúde, por exemplo), recomenda-se que a periodicidade seja trimestral.

\section{Tempo de existência}

Os periódicos que conseguem manter uma vida longa, também são bastante valorizados.

\section{Difusão}

A distribuição e divulgação devem receber uma atenção especial dos editores.

\section{Indexação}

$\mathrm{O}$ editor deve procurar indexar sua revista nas principais bases de dados de acordo com a temática do periódico. A inclusão em bases de dados que divulgam o texto completo dos artigos também acrescentam valor agregado ao periódico.

\section{Apresentação gráfica ("layout")}

Deve ser de boa qualidade a apresentação do periódico como um todo, especialmente suas ilustrações, tanto no formato impresso como no eletrônico.

\section{CONSIDERAÇÕES FINAIS}

Os editores científicos devem cuidar da qualidade global das suas publicações levando em consideração aspectos referentes a sua forma (normalização) e mérito (conteúdo) para que as mesmas atinjam uma qualidade global.

Os editores devem evitar a proliferação de revistas na mesma especialidade, dispersando fundos e esforços de seus órgãos publicadores e das agências financiadoras. Na atualidade, frente às dificuldades financeiras nos diferentes segmentos da sociedade, o compartilhamento de recursos materiais, físicos e humanos vem sendo a solução mais adequada aos editores para garantir a qualidade, a continuidade e a periodicidade de suas publicações científicas.

\section{REFERÊNCIAS}

1. Arends L. Las revistas médicas venezolanas: evaluación de sua calidad. Acta Cient Venezolana 1968; 19:148-51.

2. Braga GM, Obherhofer A. Diretrizes para a avaliação de periódicos científicos e técnicos brasileiros. Rev Lat Doc 1982; 2:27-31.

3. Castro RC, Ferreira MCG. Periódicos latino-americanos: avaliação das características formais e sua relação com a qualidade científica. Ci Inf 1996; 25:357-67.

4. Ferreira MCG. Apresentação das normas da ABNT. In: Curso de Editoração Científica: 2001; Atibaia Notas de 
Ferreira MCG, Krzyzanowski RF. Periódicos científicos: critérios de qualidade. Pesqui Odontol Bras 2003;17(Sup1 1):43-8.

aulas compiladas. Atibaia: Associação Brasileira de Editores Científicos; 2001a. p.62-71.

5. Ferreira MCG. Avaliação de periódicos científicos. In: Fórum Nacional de Padronização e Divulgação da Produção Científica: 2001; Fortaleza. Fortaleza: Universidade Federal do Ceará; 2001b.

6. Krzyzanowski RF et al. Programa de apoio às revistas científicas para a FAPESP. Ci Inf 1991; 20:137-50.

7. Krzyzanowski RF, Ferreira MCG. Avaliação de periódicos científicos e técnicos brasileiros. Ci Inf 1998; 27:165-75.

8. Martins MD. Avaliação da normalização de periódicos brasileiros nas áreas de ciência e tecnologia. Rev Bibliotecon 1986; 14:197-208.
9. Unesco. Grupo de trabajo para la selección de revistas científicas latino-americanas: 1964; Rio das Pedras, Puerto Rico. Montevideo: Centro de Cooperación Científica de la Unesco para la América Latina; 1964.

10. Wieers L. A vision on the library of the future. In: Geleijnse $\mathrm{H}$, Grootaers C (eds). Developing the library of the future: the Tiburg experience. Tiburg: Tiburg University; 1994. p.1-11.

11. Yahn VG. Avaliação de periódicos brasileiros de agricultura. Rev Bras Bibliotecon Doc 1985; 18:39-53.

12. Yamamoto $\mathrm{OH}$. Periódicos científicos em psicologia: uma proposta de avaliação. Infocapes 1999; 7:7-13. 